\title{
Harvesting Energy from Magnetic Fields to Power Condition Monitoring Sensors
}

\author{
Nina M. Roscoe and Martin D. Judd, Senior Member, IEEE
}

\begin{abstract}
Condition monitoring is playing an increasingly important role within electrical power networks, where its use can help to reduce maintenance costs, improve supply reliability and permit increased utilization of equipment capacity by providing a measure of actual operating conditions as an alternative to relying on more stringent 'worst case scenario' assumptions. In this context, energy harvesting may have a role to play in that it offers the possibility of realizing autonomous, selfpowering sensors that communicate their data wirelessly. In the vicinity of electrical transmission and distribution equipment, alternating magnetic fields at the power frequency offer a potential source of energy that does not require hard-wiring or batteries. There are many potentially useful locations for sensors where the level of magnetic flux density may be sufficient to provide enough power for a low-power wireless sensor node. This paper describes a 'free-standing' inductive harvester for use in positions where there is an ambient magnetic field due to conductors that are remote and/or inaccessible. Using data from surveys of magnetic flux density levels at two substations, optimum core and coil designs for the harvester were obtained through theoretical analysis and experiment. A demonstrator was then constructed in which a wireless sensor becomes selfpowering when immersed in a $50 \mathrm{~Hz}$ magnetic field. Laboratory results show that this system can deliver a useful average power of $300 \mu \mathrm{W}$ when placed in a magnetic flux density of $18 \mu \mathrm{T}_{r m s}$.
\end{abstract}

Index Terms - Energy harvesting, inductive coupling, magnetic field, condition monitoring, wireless sensors

\section{INTRODUCTION}

Energy harvesting $(\mathrm{EH})$ techniques are increasingly being investigated as alternatives to batteries, particularly in lowpower sensing applications [1]. Motivating factors include advances in wireless network technologies and the continual decrease in power requirements of electronic devices. Eliminating batteries has the potential to address manufacturing and disposal issues that can impact adversely on the environment. If $\mathrm{EH}$ power sources can be made sufficiently robust and reliable, there will be benefits both in terms of cost and the environment. In the context of high voltage (HV) and substation applications, the alternative of a 'tethered' power supply for sensors negates any advantage of

Manuscript received April 25th, 2012. This work was supported and funded by National Grid ref: 227819/FFR.

N.M. Roscoe is with the High Voltage Technology Research Group, University of Strathclyde, Glasgow (Corresponding author, Tel: +44 141548 2120; fax: +44 141552 5398; e-mail: nina.roscoe@eee.strath.ac.uk).

M.D. Judd is with the High Voltage Technology Research Group, University of Strathclyde, Glasgow (e-mail: m.judd@eee.strath.ac.uk). wireless sensing.

A significant body of research has shown that devices or systems requiring mean power levels of between $200 \mu \mathrm{W}$ to about $10 \mathrm{~mW}$ can be powered from a range of ambient energy sources. Energy sources such as wind [2,3] and solar [4,5] are obvious options, where power levels can be considerable. EH technologies that deliver power at lower levels include vibration (piezoelectric [6], electromechanical [7] and electrostatic [8]), thermal [9] and electromagnetic (inductive $[10,11,12]$ and capacitive $[13,14])$. $\mathrm{EH}$ is increasingly being used in industry, employing energy sources such as vibration [15], induction [16,17], thermal [18] and solar [19].

Condition monitoring (CM) is playing an increasingly important role within electrical power networks, helping to reduce maintenance costs and improve supply reliability by providing more accurate and more frequent measures of plant health. Additional sensors can help improve utilization of equipment capacity by providing knowledge of the actual operating conditions of individual system components as opposed to relying on macroscopic models or conservative estimates. However, the installation and cabling costs for conventional sensors can be a considerable barrier to their deployment. Somewhat paradoxically, given the proximity of large flows of electrical energy, power is rarely available in a convenient, low-voltage form within substations and other locations where sensors may be required. Making use of $\mathrm{EH}$ therefore seems an attractive option for powering CM sensors.

There are various location-dependent sources of harvestable energy that may be available for sensors on the electrical supply network (wind, solar, vibration, electromagnetic, etc), each of which may have a role to play. This paper focuses on harvesting energy from the ambient power-frequency magnetic fields present in $\mathrm{HV}$ substations and near power cables, busbars and transformers.

Energy from the magnetic fields surrounding $\mathrm{HV}$ conductors has been used previously to power protection relays directly from current transformers (CTs) [20]. More recently, a number of transmission line mounted self-powering (CT-based) sensors have been developed [16,17]. The housings of these devices float at $\mathrm{HV}$ potential and provide a range of wireless measurements such as line sag, current, vibration and conductor temperature. In contrast, the investigation reported here involves harvesting energy from the ambient magnetic fields without the advantage of a complete magnetic circuit enclosing the current-carrying conductor. The non-enclosing scenario has previously been studied for a harvester in close proximity to the conductor 
[12], however the field lines in close proximity to the conductor are generally on a tight radius, in contrast to field lines at distances greater than a few $\mathrm{cm}$ from the conductor, or even around energized equipment with other geometry, such as a transformer. This research addresses mounting positions where sensors could be installed safely by personnel without a power outage, for the purposes of supplying very low power wireless sensors $(\leq 500 \mu \mathrm{W})$. This means working at much greater distances from the source of the magnetic field, with a much less efficient coupling mechanism, where the magnetic flux density is relatively uniform and the field lines can be considered to be straight. Fig. 1 illustrates the differences between the conventional CT method and the approach adopted here.

Despite its coupling advantages, the CT-based harvester of Fig. 1(a) is not an ideal solution in many locations where a low-cost fully autonomous sensor could be beneficial, such as:

- Where live-line working is not permitted and currentcarrying conductors at $\mathrm{HV}$ potential cannot be de-energized to allow installation.

- On conductors lacking sufficient clearance to install a CT, e.g. cables laid in trefoil arrangement in a tunnel [21].

- At positions where the sensor cannot assume the same potential as the $\mathrm{CT}$, since any wires linking the $\mathrm{CT}$ to the sensor would compromise the HV electrical insulation.

Inductive harvesting that is not based on the use of a CT will be termed 'free-standing' within this paper. Potential applications include:

- Outdoor substations where wind or solar energy harvesters could be vulnerable to storm damage.

- Indoor substations without wind or solar power.

- On equipment with insignificant levels of vibration.

Optimization of CT-based inductive harvesters has been studied previously by the authors [22] and by Bhuiyan et al [10]. While coil design is quite different for a free-standing harvester, there is much commonality in terms of converting the raw ac coil output into a useful dc supply voltage. A voltage multiplier can boost the ac output voltage (using diodes with very low forward voltage drop to minimize losses), as well as providing ac to dc conversion [10]. The coil can be modeled as an ideal voltage source in series with its equivalent resistance and an inductance in an analogous manner to the CT-based harvester [10]. However, unlike a CT, the free-standing harvester involves a magnetic circuit with a very large air-gap. This causes a large reduction in the overall effective permeability of the magnetic circuit. The path $l_{g}$ shown in Fig. 1(b) illustrates that, for the free-standing harvester, the air gap is very large compared to the length of the core. The standard equations used to modify the permeability of a core to allow for the presence of an air-gap [23] depend on an assumption that the gap is much shorter than the path length within the core, and hence are not applicable. Instead, we have used experimental measurements to develop empirical design guidelines for free-standing harvesting coils. These have been used to design a harvester that delivers $300 \mu \mathrm{W}$ when placed in a magnetic field with flux density $B=18 \mu \mathrm{T}_{r m s}$, which proved sufficient to power a wireless temperature sensor module.

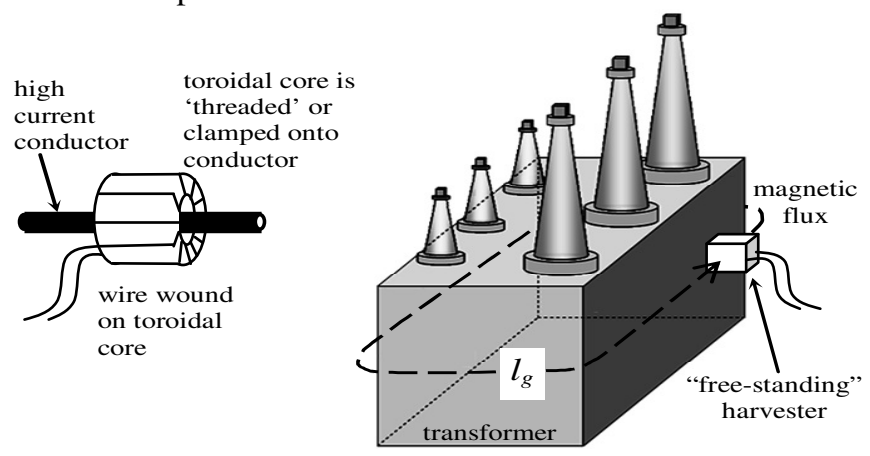

(a)

(b)

Fig. 1. Inductive harvesting: (a) CT-based harvester enclosing a conductor, (b) Free-standing harvester sitting in a magnetic field, in this example it is the magnetic field near a transformer.

\section{Survey of SubStation Flux DEnsity LEVELS}

Little published data is available for levels of $B$ in substations, other than confirmation that the flux density does not exceed regulatory levels in the 'safe areas' that personnel can access. Calculating or simulating flux density levels is impractical due to the physical complexity of the layout. Therefore, two very different $400 \mathrm{kV}$ air-insulated transmission substations were surveyed to establish realistic levels for $B$. Measurements were taken in safely accessible areas at locations near equipment (post insulators, disconnectors, wall bushings, circuit breakers) on which CM sensors might usefully be installed. $B$ was measured using both a Narda EFA 300 field analyzer [24] and an experimental magnetic field probe designed to be representative of a small energy harvesting coil. The experimental probe, pictured in Fig. 2(a), comprised 5000 turns of $0.22 \mathrm{~mm}$ diameter enameled copper wire uniformly wound onto a $228 \mathrm{~mm}$ long ferrite rod with rectangular cross-section $(25 \mathrm{~mm} \times 3.8 \mathrm{~mm})$. The core was formed from six Ferroxcube 3C90 cores [25], each $38 \mathrm{~mm}$ in length, with a nominal relative permeability of 1720. A $4 \mu \mathrm{F}$ series capacitor resonates with the $2.14 \mathrm{H}$ selfinductance of the coil to maximize output voltage at $50 \mathrm{~Hz}$ and reduce noise and interference. This capacitor also serves as the first capacitor in a Cockcroft-Walton single stage voltage multiplier that was interfaced to a voltage logger [26], as shown in Fig. 2(b). The overall probe output voltage exhibits a non-linear response, so a look-up table was used to convert between dc output voltage and $B$.

The first site to be surveyed was an outdoor substation at a node in the transmission network. Results from this survey are summarized in Fig. 3(a). The second survey took place at an indoor substation fed by a nuclear generating station. The magnetic flux density levels at this site were significantly higher, as can be seen in Fig. 3(b).

Analysis of the data in Fig. 3 indicated that $50 \%$ of the measurements at the outdoor substation lie within the range 1.7-3.9 $\mu \mathrm{T}_{r m s}$, while at the indoor substation $50 \%$ of the 
measurements lie within the range 13-59 $\mu \mathrm{T}_{r m s}$. Maximum $B$ measured near to equipment of interest was $13 \mu \mathrm{T}_{r m s}$ for the outdoor substation and $167 \mu \mathrm{T}_{r m s}$ for the indoor substation.

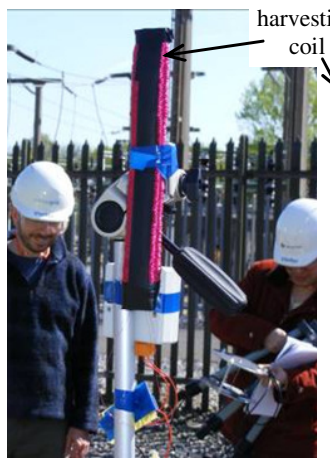

(a)

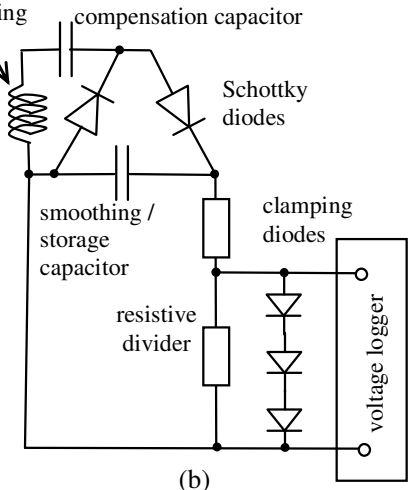

(b)
Fig. 2. Experimental probe used for measurement and logging of magnetic flux density $B$. (a) In use at a $400 \mathrm{kV}$ substation. (b) Circuit diagram.

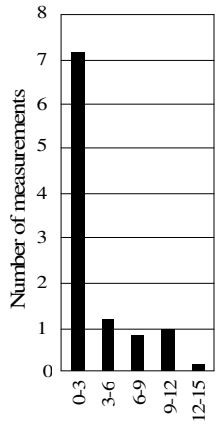

Magnetic Flux Density Range $\left(\mu \mathrm{T}_{r m s}\right)$ (a)

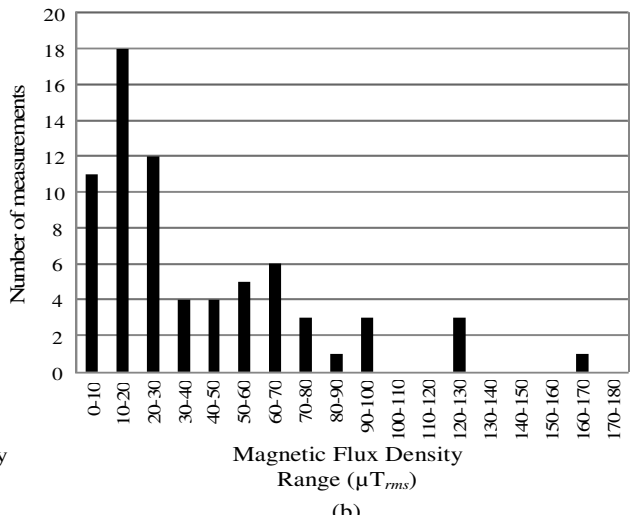

(b)

Fig. 3. Distribution of measured $B$ for all measurement locations at the outdoor (a) and indoor (b) $400 \mathrm{kV}$ air-insulated substations. The distribution of measured $B$ is displayed graphically as the number of measurements found in each range of $B$ values.

Measurements of variation in $B$ over time were also made using the experimental probe and data logger. One position in each substation was selected for longer term measurement. The outdoor substation showed a variation in $B$ between 16.5 $\mu \mathrm{T}_{r m s}$ and $6 \mu \mathrm{T}_{r m s}$ over the 2-hour period shown in Fig. 4.

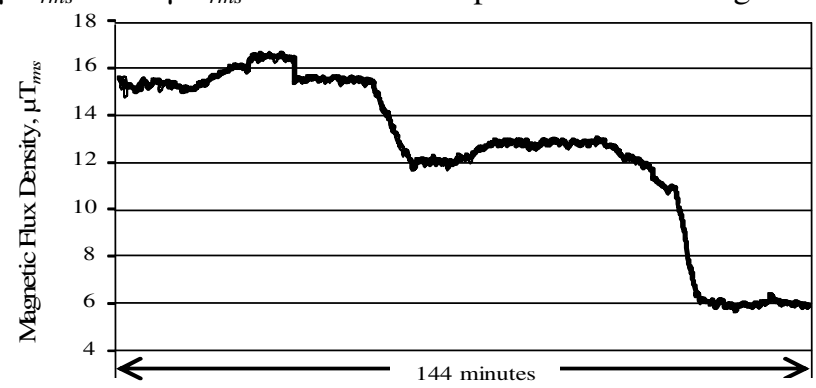

Fig. 4 Variation in $B$ as a function of time, measured in the outdoor substation.

In contrast, $B$ at the indoor substation remained steady in the range $31-32 \mu \mathrm{T}_{r m s}$. This difference was expected since the nuclear station supplies baseload power (constant current) .

\section{Design OF THE FREE-STANDING HARVESTER}

Harvesting coil size is dependent on both the available $B$ and the power consumption of the wireless sensor. Required power can be estimated using the analysis of sensor power consumption in [27], which indicates that a low power sensor might draw an average current of $80 \mu \mathrm{A}$ at $5 \mathrm{~V}(400 \mu \mathrm{W})$.

\section{A. Theory}

The open circuit voltage $V_{o c}$ developed on an $N$-turn coil wound on a cylindrical core of diameter $D$, length $l$ and relative permeability $\mu_{r}$, such as the one shown in Fig. 5, can be found using Faraday's Law (1).

$$
V_{o c}=2 \pi f \mu_{e f f} N \pi(0.5 D)^{2} B
$$

where $B$ is the magnitude of the component of magnetic flux density (in air) parallel to the coil axis and $f$ is the frequency of $B . \mu_{e f f}$ is the effective relative permeability, which lies in the range $1<\mu_{e f f}<\mu_{r}$. We define $\mu_{e f f}$ as equal to the ratio of $V_{o c}$ measured with the core in place to that measured with an air core. In cores without an air gap, $\mu_{\text {eff }}$ should be close to the inherent relative permeability of the core material. However, when the air gap is very large, there is a dramatic reduction in the open circuit voltage obtained from the coil. It has been shown previously [21] that $\mu_{e f f}$ can be calculated from measurements of coil self inductance, $L_{s}$, using

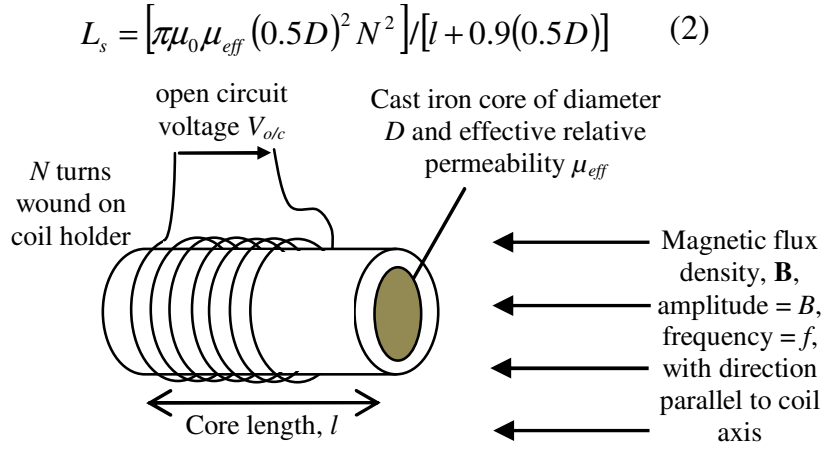

Fig. 5. Coil harvesting energy from the magnetic field. $\mu_{r}$ is the inherent relative permeability of cast iron, and $\mu_{e f f}$, is the effective permeability achieved by a cast iron core of this shape.

Maximum output power will be obtained with a matched load impedance. For a resistive load, $L_{s}$ should be compensated using a series capacitance $C_{s}=1 /\left[(2 \pi f)^{2} L_{s}\right]$ and $R_{L}$ should equal the coil series resistance $R_{s}$, as in Fig. 6. If the coil develops an open circuit voltage $V_{o c}$ in a given magnetic field and is optimally terminated, the output voltage $V_{\text {out }}$ will be half the open circuit voltage:

$$
V_{\text {out }}=0.5 V_{\text {o/c }}=\pi f \mu_{\text {eff }} N \pi(0.5 D)^{2} B
$$

Output power per unit volume $P_{\text {out }} / \mathrm{Vol} \mathrm{Wm}^{-3}$ can be found from this output voltage as follows, using the length of the core, $l$, to find core volume, $\pi(0.5 D)^{2} l$ :

$$
P_{\text {out }} / \mathrm{Vol}=\frac{\left[\pi^{2} N(0.5 D)^{2}\right]^{2}}{R_{s} \pi(0.5 D)^{2} l} \mu_{e f f}{ }^{2}(f B)^{2}
$$

When $f$ and $N$ are small, $R_{s}$ can be estimated as $R_{s}=\rho N \pi D$, where $\rho$ is the resistance per unit length of the wire, and (4) can be simplified to 


$$
P_{o u t} / V o l=\frac{\pi^{2} D N}{4 \rho l} \mu_{e f f}^{2}(f B)^{2}
$$

If the amplitude and frequency of $B$ and the wire diameter are taken as being constant in (5), the following useful relationship for output power per unit volume from a coil is obtained:

$$
P_{\text {out }} / \mathrm{Vol} \propto \frac{D N}{l} \mu_{e f f}^{2}
$$

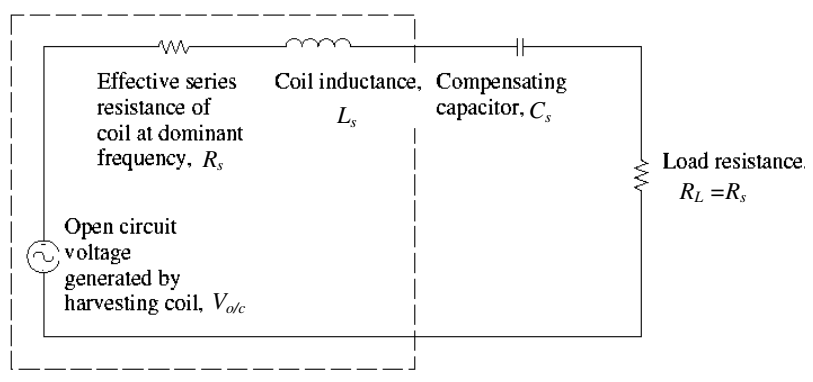

Fig. 6. Equivalent circuit of the harvesting coil with ideal matching. Coil inductance, $L_{s}$, is compensated at $50 \mathrm{~Hz}$ by the series capacitor, $C_{s}$. Load resistance $R_{L}$ is equal to coil resistance, $R_{s}$.

\section{B. Core Design}

A complication within (6) is that $\mu_{e f f}$ and the core dimensions are not independent variables. The relationship between $\mu_{e f f}$ and core length to diameter ratio, $l / D$, has been studied previously by measuring a range of coils with different core materials and sizes [21]. Results indicated that cores should preferably be long and thin and that core material has limited influence provided its inherent permeability is large compared with $\mu_{\text {eff }}$. However, this initial investigation did not establish limits to these findings, so a more complete study has been carried out. The data presented in this paper includes the measurements reported in [21] but introduces new measurements made using cast iron cores with a significantly greater range of $l / D$ ratios.

Measurements were carried out in a $50 \mathrm{~Hz} B$-field within the test apparatus shown in Fig. 7. This uses three concentric coils to create a region of uniform magnetic, as proposed by Maxwell [28]. If the centre coil has radius $a$, the two outer coils are separated from it by a distance $a \sqrt{3 / 7}$ and the radius of the outer coils is $a \sqrt{4 / 7}$. The ampere-turns product of the outer two coils should be $49 / 64$ that of the inner coil. The Maxwell coil set used for these experiments had a centre coil of radius $760 \mathrm{~mm}$, with 64 turns on the centre coil and 49 on each of the outer coils.

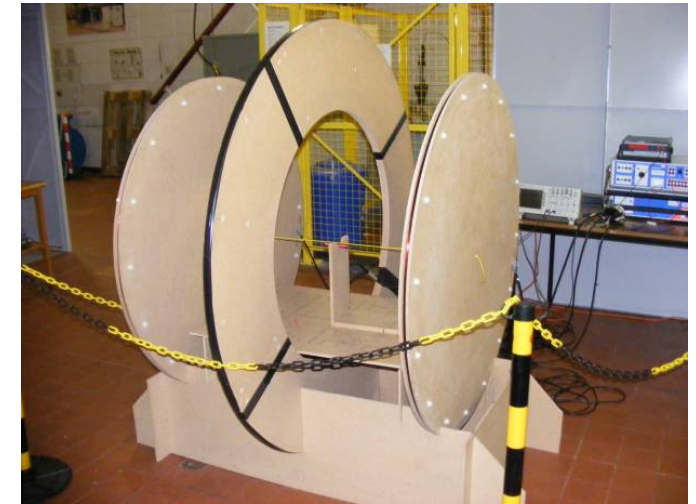

Fig. 7. Maxwell coils used to generate a uniform $B$-field in the laboratory.

Measurements were taken for four different core materials: laminated steel $\left(\mu_{r} \approx 600-2000\right) \quad[29,30]$, cast iron $\left(\mu_{r} \approx 200\right)$ [30], ferrite 3C90 $\left(\mu_{r} \approx 1720\right)$ [25] and ferrite $3 \mathrm{~F} 4$ $\left(\mu_{r} \approx 840\right)$ [25]. Coils comprising 1000 turns of $0.22 \mathrm{~mm}$ diameter copper wire were wound on each core and tests were carried out at $B=700 \mu \mathrm{T}_{r m s}$. Data from all of the core measurements is presented in Fig. 8, which suggests that, for $l / D$ less than about $10, \mu_{e f f}$ is relatively independent of core material, and is roughly proportional to $l / D$. When $l / D$ is greater than about 15 , the effective permeability of cast iron increases only very gradually with $l / D$ but the effective permeability of ferrite cores continues to increase. However, cast iron is easier to obtain in a wide range of sizes and is cheaper than ferrite. Hence, for $l / D$ ratios of less than 15 , cast iron is a suitable core material, while for larger $l / D$ values a material with higher inherent permeability should be used.

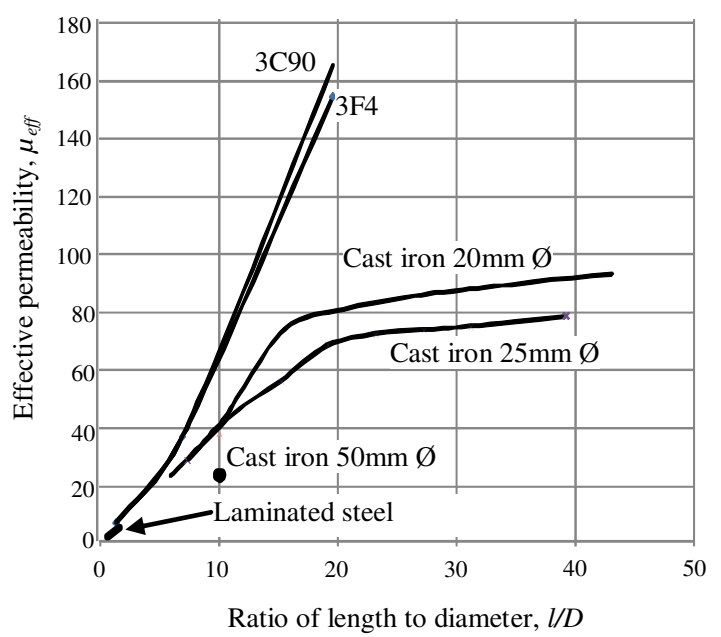

Fig. 8. Effective relative permeability as a function of length to diameter ratio for cores of different materials and diameters.

The highest $l / D$ ratio tested for cast iron was 43 . Ferrite cores were not readily available with $l / D$ greater than 3 (ferrite cores with higher $l / D$ were constructed by tightly taping individual cores end to end). Hence, it seems likely that $l / D$ will be limited to around 40 using cast iron, and to about 3 for ferrite. In any case, a very long, thin coil will be more difficult to manufacture and potentially more fragile. These issues place a practical limit on the $\mu_{e f f}$ value that can be 
achieved for the harvester.

From the empirical curves in Fig. 8, some helpful approximations can be made in order to select an optimum $l / D$ ratio. When $l / D$ is in the region where $\mu_{e f f}$ is approximately proportional to $l / D$ (cast iron with $l / D<$ 12), (6) indicates that:

$$
P_{\text {out }} / \mathrm{Vol} \propto N \mathrm{~N} / \mathrm{D}
$$

Similarly, when $l / D$ is in the region where $\mu_{\text {eff }}$ for cast iron is relatively insensitive to changes in $l / D$, the relationship can be approximated as:

$$
P_{\text {out }} / \mathrm{Vol} \propto \mathrm{DN} / \mathrm{l}
$$

Equation (7) suggests that $l / D$ should be maximized for maximum $P_{\text {out }} / \mathrm{Vol}$. However, (8) shows that if $l / D$ is increased further, $P_{\text {out }} / \mathrm{Vol}$ will tend to decrease again. Hence maximum $P_{\text {out }} / \mathrm{Vol}$ is expected to occur around the transition region where the relationship between $l / D$ and $\mu_{e f f}$ changes from approximately linear to approximately constant. For cast iron, this point occurs for $l / D \approx 12-16$. While (7) and (8) involve considerable simplification, they provide a basis for selecting an optimal value for $l / D$.

\section{Coil Design}

For practical reasons, the length of the coil was limited to $0.5 \mathrm{~m}$ for this harvester design. A cast iron rod of diameter 50 $\mathrm{mm}$ was selected, giving an $l / D$ value of 10 , which is close to the range of optimum ratio of 12-16. The influence of the number of turns on harvester performance was then investigated experimentally. For a fixed $l / D$, (6) suggests that output power per unit volume should increase linearly with the number of turns wound on the core. In practice, output power does not continue to increase with $N$ due to the effects of eddy currents in the core and distributed impedance effects, which inflate the coil series resistance when $N$ is large.

The series resistance generated by eddy currents has been previously examined for a coil wound on a rectangular laminated iron core [31]. A similar method can be used to analyze the cylindrical core by carrying out an analysis in the cylindrical co-ordinates of Fig. 9 and integrating for a series of concentric thin cylinders instead of thin flat sheets. The use of concentric cylinders is appropriate because the magnetic field is aligned with the core axis (see Fig. 5), generating eddy currents that circulate in the $\theta$-axis.

Using the method outlined in [31], the magnetic field inside the core at distance $r$ from its axis can be shown to be:

$$
H_{c}(r)=H_{c o} \cosh \left(\frac{1+j}{\delta_{t}} r\right) / \cosh \left(\frac{1+j}{\delta_{t}} 0.5 D\right)
$$

where $H_{c o}$ is the magnetic field at $r \rightarrow 0.5 D$ and $\delta_{t}=\sqrt{\rho_{\text {iron }} /\left(\pi \mu_{0} \mu_{\text {eff }} f\right)}$, in which $\rho_{\text {iron }}$ is the resistivity of the core material. Approximating the coil as an infinite solenoid carrying current $I$, standard electromagnetic theory indicates that $H_{c o}=N I / l$. Incremental flux for a single cylinder is found by multiplying (9) by the flux linkage area $2 \pi r d r$, giving:

$$
\Phi_{c}(r)=\mu_{0} \mu_{e f f} H_{c o} \frac{\cosh \left(\frac{1+j}{\delta_{t}} r\right)}{\cosh \left(\frac{1+j}{\delta_{t}} 0.5 D\right)} 2 \pi r d r
$$

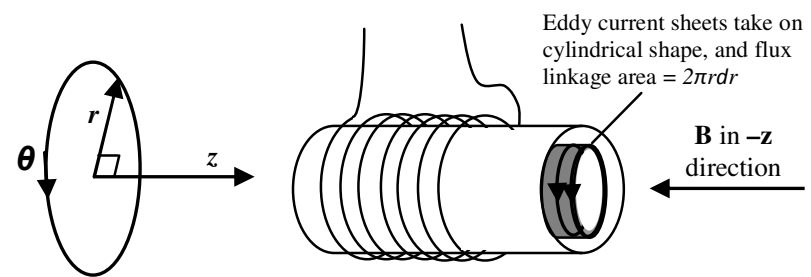

Fig. 9. Cylindrical co-ordinate system and eddy current "cylinders"

Total flux linking with the coil is determined by integrating (10) between the limits $r=0$ and $r=0.5 D$ :

$$
\Phi_{c}=\frac{2 \pi \mu_{0} N^{2} I / l}{\cosh \left(\frac{1+j}{\delta_{t}} 0.5 D\right)} \int_{0}^{0.5 D} r \mu_{e f f} \cosh \left(\frac{1+j}{\delta_{t}} r\right) d r
$$

Making an assumption that $\mu_{\text {eff }}$ is constant for all $r$, then the total flux linkage is given by:

$$
\Phi_{c}=\frac{\delta_{t}}{1+j} \frac{\pi \mu_{0} \mu_{e f f} N^{2} I / l}{\cosh \left(\frac{1+j}{\delta_{t}} 0.5 D\right)}\left\{D \sinh ^{\frac{1+j}{\delta_{t}} 0^{.5 D}}+\frac{2 \delta_{t}}{1+j}\left(1-\cosh ^{\frac{1+j}{\delta_{t}} 0^{-5 D}}\right)\right\}
$$

Coil impedance is found from total flux linkage [31] using $V=j \omega \Phi_{c}$ and then dividing this induced voltage by the coil current, $I$ :

$$
Z=\frac{j \omega \delta_{t}}{1+j} \frac{\pi \mu_{0} \mu_{e f f} N^{2} / l}{\cosh \left(\frac{1+j}{\delta_{t}} 0.5 D\right)}\left\{D \sinh ^{\frac{1+j}{\delta_{t}} 0.5 D}+\frac{2 \delta_{t}}{1+j}\left(1-\cosh ^{\frac{1+j^{\prime}}{\delta_{t}} 0.5 D}\right)\right\}
$$

Taking the real part of (13) gives the core loss series resistance. Distributed coil impedance effects were included by using Matlab to model the coil as a transmission line made up of $N$ elements. Each element consisted of a series resistance and inductance, and a shunt capacitance to ground. For each element, the series inductance was found from total coil inductance divided by $N$, series resistance was found from the sum of copper and core loss resistances divided by $N$, shunt capacitance to ground was found from the total capacitance from one layer to the core, divided by the number of turns in a single layer (capacitance from any layer of turns to the core can be considered to be equal because the interlayer capacitance was much larger than the capacitance from the inside layer to the core). The model was used to calculate the real and imaginary parts of the coil impedance at $50 \mathrm{~Hz}$, including eddy current loss, copper loss and distributed impedance effects.

The real part of the coil impedance at $50 \mathrm{~Hz}$ can be determined empirically by placing the coil in the $50 \mathrm{~Hz}$ magnetic field and trying a wide range of series capacitance 
values, $C_{s}$, in combination with a wide range of load resistances, $R_{s}$. The combination of values of $C_{s}$ and $R_{s}$ that provides optimum impedance matching conditions will yield the greatest power, allowing the effective inductance and resistance of the coil to be estimated. Effective inductance of the coil is found from

$$
L_{s}=1 /\left[(2 \pi f)^{2} C_{s}\right]
$$

Measured and calculated values for coil effective series resistance are compared in Fig. 10 below for the real part of coil impedance as a function of $N$. Allowing for the potential inaccuracy introduced by assuming that $\mu_{\text {eff }}$ is constant for all $r$, the correlation in Fig. 10 confirms that coil resistance is dominated by eddy currents at these large values of $N$ and that (12) allows this resistance to be estimated to an accuracy well within an order of magnitude.

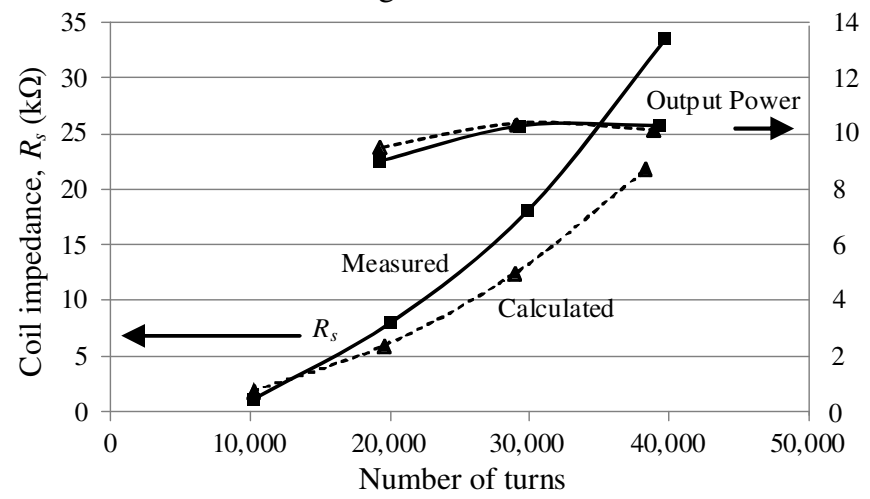

Fig. 10. Comparisons between measured (solid line) and calculated (dashed line) values for coil effective series resistance $\left(R_{s}\right)$ and output power $\left(P_{\text {out }}\right)$ for cast iron core with $l=0.5 \mathrm{~m}, D=50 \mathrm{~mm}, f=50 \mathrm{~Hz}$ and $B=66 \mu \mathrm{T}_{r m s}$.

Measured output power under optimum impedance matching conditions was plotted for 20,000, 30,000 and 40,000 turns on the coil, and is also compared with calculated output power in Fig. 10. Output power was calculated using (4), with $B=66 \mu \mathrm{T}_{r m s}, D=0.05 \mathrm{~m}, l=0.5 \mathrm{~m}, \mu_{\text {eff }}=23, f=50$ $\mathrm{Hz}$, and $R_{s}$ values as shown in Fig. 10.

\section{Converting ac output to regulated dc voltage}

The single stage Cockcroft-Walton doubler in the circuit shown in Fig. 11 was used to convert the ac harvester output voltage to dc. This is an efficient means of converting from ac to dc while simultaneously boosting voltage; a technique recognized as being efficient for self-powering condition monitoring sensors where harvested voltages can be quite low [10,21]. A dc to dc converter was used to regulate the dc output voltage from the doubler. The storage capacitor provides an energy reserve for short periods of low $B$ and serves as the smoothing capacitor for the doubler circuit. The diodes for the doubler were Schottky diodes selected for low conduction loss. A Zener diode ensures safe dissipation of excess energy when high $B$ would otherwise lead to overvoltage on the electronics.

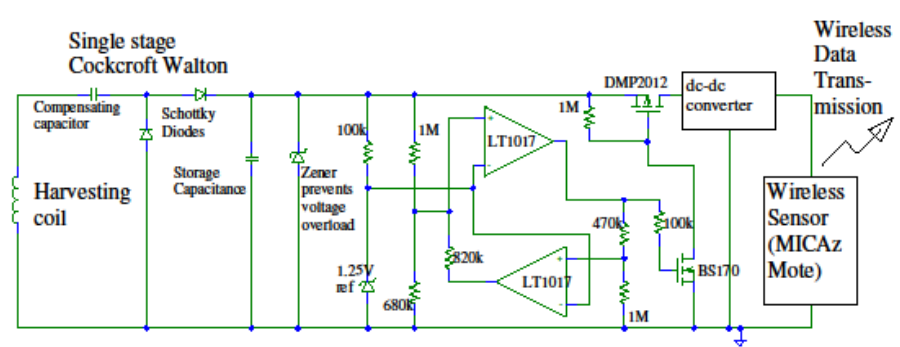

Fig, 11. Complete free-standing inductive harvester circuit used for selfpowered transmission of temperature data across the laboratory. The dc-dc converter is a MAX639, the low leakage Zener diode limits at $12 \mathrm{~V}$ and the storage capacitor is $6.8 \mathrm{mF}$ Aluminum electrolytic.

\section{E. Wireless Sensor}

A MICAz (MPR2400) $2.4 \mathrm{GHz}$ Mote module [32] was chosen for laboratory trials because it is designed for development of wireless sensor platforms, and hence is versatile and easily configurable. This module requires approximately $0.3 \mathrm{~mW}$ average power $(90 \mu \mathrm{A}$ at $3.3 \mathrm{~V})$ when configured to transmit a temperature reading every 4 minutes. Since magnetic flux can vary with time, as shown in Fig. 4, any sensor powered by inductive energy harvesting must adapt its activity to the available energy. A buffer circuit achieves this by maintaining the sensor device in the off mode until sufficient energy has been accumulated in the storage capacitor to allow a regulated supply to be maintained to the sensor electronics for the complete duration of its measurement cycle. The buffer circuit in Fig. 11 comprises the very low supply current comparator (LT1017), a 1.25 V reference (LT1034) and two MOSFET switches (DMP2012, BS170). The buffer connects the voltage doubler to the dc to dc converter once the voltage on the storage capacitor reaches $4.6 \mathrm{~V}$ and ensures that the voltage is disconnected if the voltage on the storage capacitor drops back down to $2.1 \mathrm{~V}$. At times of low magnetic flux density, measurements would become less frequent or may cease altogether. This kind of behavior is common to many devices powered from energy harvesting and must be taken into account when determining the suitability of energy harvesting as a power source for specific applications.

The buffer circuit dissipated approximately $0.09 \mathrm{~mW}$, giving an efficiency of about $77 \%$. The electronic circuit presented a load impedance of approximately $6 \mathrm{k} \Omega$ to the harvesting coil. Since the optimum load impedance for this coil is $33 \mathrm{k} \Omega$ (see Fig. 10), this mismatch leads to an efficiency of $73 \%$. The MAX 639 achieves an efficiency of around $75 \%$ with $100 \mu \mathrm{A}$ load current and reverse diode leakage accounts for around $50 \mu \mathrm{W}$ of dissipation. Combining the efficiencies of the impedance match, diodes and the dc to dc converter leads to an overall figure of $36 \%$. To ensure delivery of $0.3 \mathrm{~mW}$ to the MICAz module therefore requires the harvesting coil to deliver about $833 \mu \mathrm{W}$, which leads to a minimum value of $B=$ $18.5 \mu \mathrm{T}_{r m s}$ using (4) for a coil with $D=0.05 \mathrm{~m}, N=40,000, R_{S}$ $=33 \mathrm{k} \Omega, \mu_{e f f}=23$ and $f=50 \mathrm{~Hz}$.

\section{RESULTS AND DISCUSSION}

The inductive harvester demonstrator shown in Fig. 12 was 
tested in the Maxwell coils apparatus (Fig. 7). The MICAz wireless sensor was configured to transmit a temperature measurement every 4 minutes and this data was received and displayed on a laptop PC. At this transmission rate, the MICAz power consumption is dominated by quiescent current. Transmission power is effectively negligible, and therefore this is the lowest possible MICAz operational energy consumption, at a value of $0.3 \mathrm{~mW}$. The minimum value of $B$ at which the harvester could provide sufficient energy to the MICAz was found to be $18 \mu \mathrm{T}_{r m s}$ which agrees closely with the figure of 18.5 $\mu \mathrm{T}_{r m s}$ predicted in section III. At $18 \mu \mathrm{T}_{r m s}$ the transmission distance was $12 \mathrm{~m}$. A transmission distance of 17 $\mathrm{m}$ was achieved in a magnetic flux density of $210 \mu \mathrm{T}_{r m s}$. The greatest possible transmission distance in the laboratory is limited to $17 \mathrm{~m}$ by a combination of layout and screened walls.

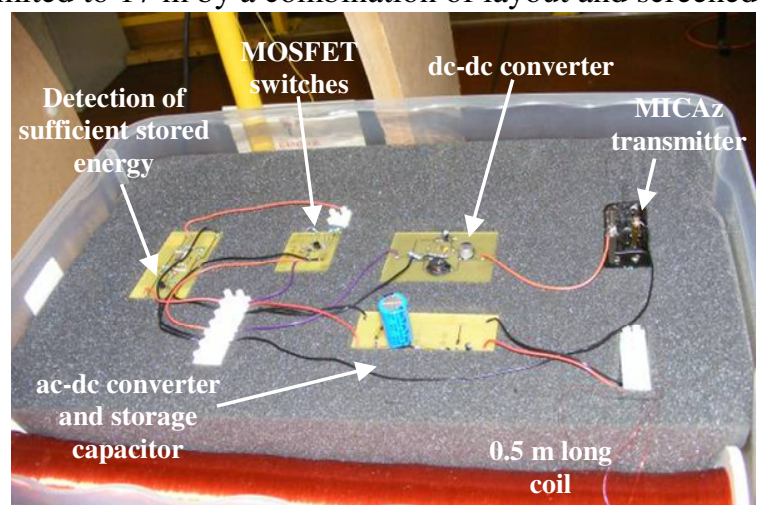

Fig, 12. Free-standing inductive harvester demonstrator using 40,000 turn coil wound on $0.5 \mathrm{~m}$ long cast iron core, $50 \mathrm{~mm}$ diameter, connected as shown in Fig. 11.

This quite large increase in $B$ required for a modest increase in transmission distance is caused by the output voltage on the dc-dc converter being set a little too high for the MICAz. Subsequent experiments have shown that a drop in voltage from $3.3 \mathrm{~V}$ to $3 \mathrm{~V}$ halves the MICAz quiescent current. The excess current consumption at higher voltage leads to discharge of the storage capacitor, causing the supply voltage to the MICAz to drop to $2.6 \mathrm{~V}$ (which is below the rated voltage range) and this restricts the transmission distance by compromising the performance of the analogue RF transmitter. Experiments on a new demonstrator have shown that this can be solved by setting the MICAz supply voltage to $2.95 \mathrm{~V}$.

When exposed to $B$ of less than $18 \mu \mathrm{T}_{r m s}$, the quiescent current requirement of the MICAz exceeds the available energy from the harvester and the buffer circuit ensures a clean shut down. Further investigation of the relationship between $B$ and transmission distance was not carried out because this relationship is very specific to the MICAz, which is a development module. Alternative wireless systems are under investigation for use in future harvesters.

The output power per unit volume can be calculated by calculating the ratio between the power delivered to the wireless module $(0.3 \mathrm{~mW})$ and the volume of the demonstrator components. The approximate dimensions of the coil plus electronics were $5 \times 8 \times 50 \mathrm{~cm}$, giving a volume of $2000 \mathrm{~cm}^{3}$. The output power per unit volume achieved is therefore approximately $1.5 \times 10^{-4} \mathrm{~mW} / \mathrm{cm}^{3}$ when exposed to $18 \mu \mathrm{T}_{r m s}$.

At a distance $4 \mathrm{~cm}$ from a conductor carrying 400 A the magnetic flux density would be around $2000 \mu \mathrm{T}_{r m s}$, using $B=\frac{\mu_{0} I}{2 \pi r}$ with $I=400 \mathrm{~A}, r=4 \mathrm{~cm}, \mu_{o}=4 \pi \times 10^{-7} \mathrm{Hm}^{-1}$. The volume of the core used for this research was $981 \mathrm{~cm}^{3}$. The output power per unit volume for this harvester would then be $1.8 \mathrm{~mW} / \mathrm{cm}^{3}$ when used $4 \mathrm{~cm}$ from a conductor carrying 400 A, since the output power per unit volume is proportional to the square of the magnetic flux density. This can be favorably compared to the compact I-core harvester which has a maximum predicted output power of around $0.7 \mathrm{~mW} / \mathrm{cm}^{3}$ at a distance of $4 \mathrm{~cm}$ from a conductor carrying 400 A [12].Better design for lower core loss and improved impedance match have the potential to further improve the device efficiency.

\section{COMPARING HARVESTING SOLUTIONS}

The incomplete magnetic circuit with which these freestanding inductive harvesters must operate lowers their efficiency when compared to an enclosing harvester. For example, the OTLM unit should achieve an output power per unit volume of $1.43 \mathrm{~mW} / \mathrm{cm}^{3}$ when mounted on a conductor carrying $65 \mathrm{~A}_{r m s}$ [17], compared to $0.1 \mathrm{~mW} / \mathrm{cm}^{3}$ generated by this free-standing harvester when mounted $4 \mathrm{~cm}$ from a conductor carrying the same current. The free-standing harvesters are therefore appropriate for situations where the conductor cannot be enclosed for practical reasons, examples of which have been given in Section I.

When attempting to make comparisons between inductive energy harvesters and other forms of energy harvesting, it is difficult to establish a meaningful basis for the assessment. Using datasheet graphs, a commercial vibration energy harvester (Perpetuum PMG FSH, [15]) needs $0.006 \mathrm{~g}_{r m s}$ to generate $0.3 \mathrm{~mW}$, giving $0.001 \mathrm{~mW} / \mathrm{cm}^{3}$. A representative thermoelectric device (Micropelt MPG-D651, [18]) delivers $0.3 \mathrm{~mW}$ for a temperature difference of $10^{\circ} \mathrm{C}$ with an aircooled heatsink, corresponding to $1.6 \mathrm{~mW} / \mathrm{cm}^{3}$. Solar energy delivers around $0.03 \mathrm{~mW} / \mathrm{cm}^{3}$ [19] for light levels in the region of 200 lux [19]. While it would be helpful if these power densities could be used to select the "best" form of energy harvesting, the calculations do not take account of the critical influence of the power density of the input energy source that is being harvested. In practice, it is more likely that the choice of technology will be governed by the predominant energy source available at a given sensor location.

\section{CONCLUSIONS}

Magnetic flux density $B$ has been measured in two representative transmission substations, showing that in some substations there is sufficient $B$ for inductive harvesting.

A free-standing inductive harvester concept has been presented. Optimum coil design for this harvester has been explored using theoretical analysis, which was subsequently verified by a range of experimental measurements. A length to diameter ratio in the region from 12-16 was found to be 
optimum for a cylindrical core, with cast iron providing the best compromise between practicality, economy and performance, leading to significantly greater efficiency than previously demonstrated in the literature.

A free-standing harvester has been used to demonstrate a fully self-powered wireless temperature sensor in the laboratory, using only the energy from a low-level $50 \mathrm{~Hz}$ magnetic field. Temperature measurements were transmitted to a PC-based receiver over a range of $12 \mathrm{~m}$ every 4 minutes at the minimum operating level of $B=18 \mu \mathrm{T}_{r m s}$. The harvester demonstrator also contained a buffer circuit to ensure that operation begins only when sufficient $B$ is available, and implements complete shutdown if $B$ falls below the minimum value required for operation. The next phase of this work will involve developing a ruggedized self-powered wireless temperature sensor for trials and long-term deployment in the substation environment.

\section{ACKNOWLEDGMENTS}

This research was funded by National Grid (project ref. 227819/FFR). The authors are grateful to Mr. Carl Johnstone and Mr. Leigh Fraser of National Grid for providing substation access. Thanks are also due to Mr. Frank Cox of Strathclyde University, who constructed the harvester and many test coils during its development, and to Dr Peter Baker, who provided advice and support in relation to the wireless sensors.

\section{REFERENCES}

[1] C. Kompis and S. Aliwell (Eds), "Energy Harvesting Technology to Enable Remote and Wireless Sensing," Sensors and Instrumentation Knowledge Transfer Network, June 2008.

[2] M. A. Weimer, T. S. Paing, and R. A. Zane, "Remote area wind energy harvesting for low-power autonomous sensors," Power Electronics Specialists Conference, 2006, pp 1-5

[3] Y.K. Tan and S.K. Panda, "Self-Autonomous Wireless Sensor Nodes With Wind Energy Harvesting for Remote Sensing of Wind-Driven Wildfire Spread," IEEE Transactions on Instrumentation and Measurement, Vol. 60, No. 4, April 2011, pp. 1367-1377.

[4] Vijay Raghunathan; A. Kansal, J. Hsu, J. Friedman, Mani Srivastava, "Design considerations for solar energy harvesting wireless embedded systems," Information Processing in Sensor Networks, 2005, pp. 457462

[5] S. Bader, B. Oelmann, "Enabling Battery-Less Wireless Sensor Operation Using Solar Energy Harvesting at Locations with Limited Solar Radiation," $4^{\text {th }}$ Int. conf. on Sensor Technologies and Applications (SENSORCOMM), July 2010 pp. 602-608

[6] G.K Ottman, H.F Hofmann, A.C. Bhatt, G.A. Lesieutre, "Adaptive piezoelectric energy harvesting circuit for wireless remote power supply," IEEE Trans. Power Electronics, Vol. 17, Issue: 52002 , pp. 669-676

[7] C.R. Saha, T. O'Donnell, H. Loder, S. Beeby, J. Tudor, "Optimization of an Electromagnetic Energy Harvesting Device," IEEE Trans. Magnetics, Vol. 42, Issue: 10, 2006 , pp. 3509-3511

[8] E. O. Torres and G.A. Rincón-Mora, "Electrostatic Energy-Harvesting and Battery-Charging CMOS System Prototype," IEEE Trans. On Circuits and Systems, vol. 56, No. 9, Sep 2009, pp. 1938-1948

[9] C. Lu, S.P. Park, V. Raghunathan, K. Roy, "Analysis and design of ultra low power thermoelectric energy harvesting systems," ACM/IEEE International Symposium Low-Power Electronics and Design (ISLPED), 2010, pp. 183-188

[10] R.H. Bhuiyan, R.A. Dougal, M. Ali, "A Miniature Energy Harvesting Device for Wireless Sensors in Electric Power System," IEEE Sensors Journal, Vol. 10, Issue: 7, 2010 , pp. 1249-1258
[11] T. Taithongchai, E. Leelarasmee, "Adaptive electromagnetic energy harvesting circuit for wireless sensor application," Electrical Engineering/Electronics, 6th International Conference on Computer, Telecommunications and Information Technology, 2009, Vol. 1, pp. 278-281

[12] Moghe, R.; Yi Yang ; Lambert, F. ; Divan, D. "Design of a Low Cost Self-Powered Stick-on Current and Temperature Wireless Sensor for Utility Assets," Energy Conversion Congress and Exposition (ECCE), 2010 IEEE, 12-16 Sept. 2010, pp. 4453 - 4460

[13] M. Zhu, M.D. Judd, P.J. Moore, "Energy Harvesting in Substations for Powering Autonomous Sensors," 3rd International Conference on Sensor Technologies and Applications, 2009, pp. 246-251

[14] H. Zangl, T. Bretterklieber, G. Brasseur, "Energy Harvesting for Online Condition Monitoring of High Voltage Overhead Power Lines," Instrumentation and Measurement Technology Conference Proceedings, 2008, pp. 1364-1369

[15] Perpetuum PMG FSH Free Standing Vibration Harvester, http://www.perpetuum.com/fsh.asp, Accessed Jan 2013

[16] USi Inductive Harvester, Power Donut2, http://www.usi-power.com, Accessed Jan 2013

[17] OTLM Overhead Transmission Line Monitoring system, OTLM 7100, http://www.otlm.eu/en/otlm device, Accessed Jan 2013

[18] Thermopelt MPG-D651 from Micropelt, http://www.micropelt.com/products/thermogenerator.php, Accessed Jan 2013

[19] Enocean 868MHz Solar Sensor Module, ECS310, http://www.enocean.com/en/enocean_modules/energy-harvester, Accessed Jan 2013

[20] C. Preve, "Protection of Electrical Networks," ISTE Ltd., ISBN 978-1905209-06-04, 2006.

[21] N.M. Roscoe, M.D. Judd and L. Fraser "A Novel Inductive Electromagnetic Energy Harester For Condition Monitoring Sensors", Proceedings of the 2010 International Conference on Condition Monitoring and Diagnosis, Sepetember 6-11, 2010, pp 615-618

[22] N.M Roscoe, M.D. Judd, J. Fitch, "Development Of Magnetic Induction Energy Harvesting For Condition Monitoring," Proc. of the $44^{\text {th }}$ International Universities Power Engineering Conference (UPEC), 2009

[23] "Handbook of Transformer Design and Applications", $2^{\text {nd }}$ ed. New York:McGraw-Hill, 1993.

[24] Narda Magnetic Field Analyser, EFA-300, http://www.nardasts.us/pdf_files/DataSheets/EFA200-300_DataSheet.pdf, Accessed Jan 2013

[25] Ferroxcube $3 \mathrm{C} 90$ and $3 \mathrm{~F} 4$ ferrite cores, datasheets available at www.ferroxcube.com, Accessed Jan 2013

[26] Zeta-tec Voltage Logger, Data Logger Model 4V (ZT-1003), http://www.zeta-tec.co.uk/Voltage-data-logger-serial.htm, Accessed Jan 2013

[27] M Zhu, P Baker, N M Roscoe, M D Judd and J Fitch, "Alternative Energy Sources for Autonomous Sensors in High Voltage Plants," Proc. 29th IEEE Electrical Insulation Conference (EIC 2009), June 2009.

[28] J. Clerk-Maxwell, Treatise on Electricity and Magnetism. Oxford: The Clarendon Press. ISBN 0-486-60636-8, 1873, Page 319.

[29] H.A. Toliyat, G.B. Kliman, M. Dekker, Handbook of Electric Motors. 2004, ISBN 0-8247-4105-6, Page 201.

[30] W. Bolton Engineering Science. Elsevier Newnes, ISBN 13: 978-07506-8083-7, 2006, Page 174.

[31] G. Grandi, M. K. Kazimierczuk A. Massarini, U. Reggiani and G. Sancineto, "Model of Laminated Iron-Core Inductors for High Frequencies," IEEE Trans. on Magnetics, Vol. 40, No. 4, July 2004, pp. $1839-1845$

[32] MICAz wireless sensor, (MPR2400) $2.4 \mathrm{GHz}$ Mote, http://www.memsic.com/products/wireless-sensor-networks/wirelessmodules.html, Accessed Jan 2013 